\title{
Evaluation of Data Preprocessing TechniQues FOR ANOMALY DETECTION SYSTEMS IN INDUSTRIAL CONTROL SYSTEM
}

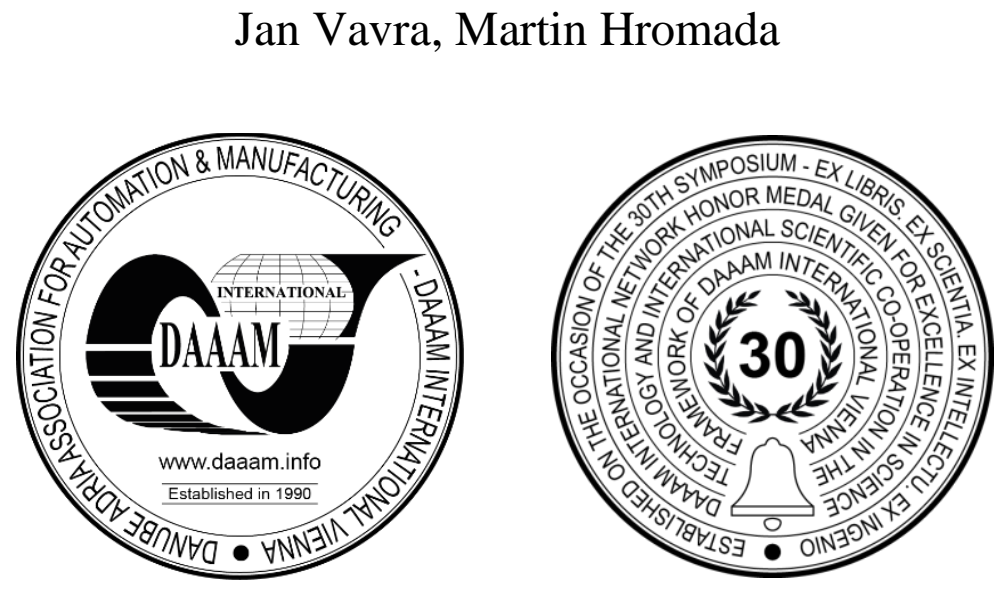

This Publication has to be referred as: Vavra, J[an] \& Hromada, M[artin] (2019). Evaluation of Data Preprocessing Techniques for Anomaly Detection Systems in Industrial Control System, Proceedings of the 30th DAAAM International Symposium, pp.0738-0745, B. Katalinic (Ed.), Published by DAAAM International, ISBN 978-3-902734-22-8, ISSN 1726-9679, Vienna, Austria

DOI: $10.2507 / 30$ th.daaam.proceedings.101

\begin{abstract}
The critical infrastructure can be defined as main cornerstone of modern society. Therefore, the cyber protection of critical systems like industrial control systems is vital for every modern state. However, conventional techniques are often ineffective to protect these systems. Thus, machine learning is an exceptional way to ensure cyber security in the case of critical infrastructure. The machine learning can process high dimension datasets with thousands of record in real-time. However, these datasets have to be in a proper format. The data preprocessing is a crucial stage in machine learning and can negatively influence final results. We introduce a comprehensive comparison of the main data preprocessing techniques in the relation of the network anomaly detection system. Moreover, the preprocessing of continuous datasets is considered as the subject of the research The neural network autoencoder is considered as an anomaly detection algorithm which is used to evaluate proposed solutions.
\end{abstract}

Keywords: Data Preprocessing; Cyber security; Machine Learning; Industrial Control System; Anomaly Detection.

\section{Introduction}

Technology has become an integral part of contemporary society. The current transition from an industrial society to the information society is accompanied by the implementation of new technologies in every part of human activity. Increasing pressure to apply ICT in critical infrastructure areas creates new vulnerabilities. Traditional safety approaches are becoming ineffective. From this perspective, the use of artificial intelligence is another evolutionary step that provides robust solutions for extensive and sophisticated systems. This paper focuses on the field of cyber security research for industrial control systems that are widely used in critical infrastructure. Within these systems, each downtime can be defined as a potential threat to the core services needed to run the company. Cybernetic protection for industrial control systems is one of the most critical types of security for a modern state. The application of machine learning has multiple usages to solve daily real-life problems as can be seen in publications [1] or [2].

Stouffer et al. [3] point to the historical evolution of ICS development. Most of the current ICS were developed without consideration of cyber security and Industry 4.0. Therefore, a considerable number of ICS devices are designed with 
security flaws. Moreover, these commonly used technologies are interconnected with traditional information and communication technologies. Therefore recently closed system has become open. This development leads to the creation of new and unexpected vulnerabilities.

The network anomaly detection system based on a neural network with autoencoder architecture was created for the purpose of the article. This anomaly detection system can be classified into a group of novelty detection techniques, also known as outlier detection or semi-supervised anomaly detection. This area of machine learning is a combination of supervised learning and unsupervised learning techniques. The novelty detection creates a classification model based on standard data without any signs of cyber attacks. The models are used to check new data, where every deviation from normal behavior is classified as an anomaly; and therefore, possible cyber attack. This field of machine learning algorithms is outlined in a considerable number of publications as Rajendran et al. [4], Ruff et al. [5], Camacho et all [6].

The data preprocessing is a vital part of machine learning, which can have a considerable impact on the final result of machine learning models. This area of interest is also highly reflected in the field of data mining. García et al. [7] dedicated the book to preprocessing of data. They introduced a considerable number of techniques. They also pointed out on shortcomings of real-world data which are negatively influenced by numerous factors. Thus, extensive data preprocessing is needed. Moreover, García et al. in the publication. [8] Moreover, Davis and Clark [9] introduced a comprehensive study of data preprocessing for network anomaly detection systems. However, this review is mainly focused on feature reduction and feature selection which is not the topic of this article. Up to our knowledge, there is no article which dealt with the analysis of preprocessing techniques for continues data in case of anomaly detection in the ICS environment. The main goal of the article is aimed to evaluate the effectiveness of data preprocessing techniques in connection to a network anomaly detection system. The evaluation criteria are Accuracy, F1 Score, False positive rate and time needed to make a prediction by the model.

The rest of the article is organized as follows. Section II introduces fundamental information about neural networks with an autoencoder architecture. The data preprocessing techniques are analyzed in Section III. In Section IV, the methods of the research are described. The next Sections (V and VI) include the results of the study and a discussion of the article.

\section{Autoencoder}

We consider autoencoder as the unique architecture of the neural network in this case. We consider autoencoder as the unique architecture of the neural network in this case. The autoencoders are designed to encode and decode of the data. The architecture of autoencoder is symmetrical with "bottleneck" in the middle. Moreover, the autoencoder learns the data representation in the process. We need to solve the regression task in case of autoencoders. Therefore, the interpretation system must be established in order to identify the anomaly. This system is described in the Methods section in detail.

The philosophy of neural network is based on nerve center in biological organisms. Neurons themselves are individual cells for storing and transmitting information necessary for the proper functioning of the organism. Rosenblatt laid the foundation of the modern neural network in his publication [4], where perceptron is introduced. The matrix operations are considered as necessary procedures within each neuron (1). The multiplication of each input $\mathrm{x}_{\mathrm{i}}$ (data record) matrix of weights $\mathrm{w}_{\mathrm{i}}$ is an essential operation for each neuron. Moreover, the weights of a neuron are a measure of the significance of the input. The $\mathrm{b}$ represents the threshold (bias) for the neuron and allows greater model variability to match the dataset.

$$
\mathrm{Y}=A\left(\sum_{i=1}^{n} x_{i} \cdot w_{i}+b\right)
$$

The threshold, together with weights and inputs, creates the internal potential of the neuron. This potential is based on biological neurons which after receiving information, excite and transmit the information otherwise remain idle. The decision of whether the neuron will be activated or not depends on the activation function (A), more precisely on the internal potential of the neuron.

$$
\mathrm{A}(\mathrm{x})=\frac{1}{1+e^{-x}}
$$

However, neural network learning is an iterative process that can be defined as an optimization task. Thus, the "Backpropagation" algorithm is used to training from errors. This algorithm first compares the actual result ( $\mathrm{Y}_{\text {real. }}$ ) With the result provided by (predicts) the model ( $\mathrm{Y}_{\text {predik. }}$ ). One of the basic algorithms used to calculate the error is the Mean Squared Error or MSE (3). The MSE is based on the difference in the mean values of the observed result. The smaller the number is, the more accurate the model. This calculation gives us the so-called loss function, which expresses how well the model matches the data for training. 


$$
\operatorname{MSE}=\frac{1}{2} \sum_{i=1}^{n}\left(Y_{i}^{\text {real. }}-Y_{i}^{\text {predik. }}\right)^{2}
$$

The Back-propagation algorithm uses MSE as a function for optimization. In this case, the MSE value is considered as an error function that must be minimized. For this reason, the optimization algorithm uses the calculated error and propagate it backward. From the output layer to the input layer while correcting the weights and thresholds of the individual neurons. Partial derivatives of the error function calculate this process according to the different weights (4), where $f$ represents the error function, and $w$ represents the weights of the $i$-th neuron in the $j$-th layer. The necessary prerequisite for fulfilling this function is its convergence to the global maximum.

$$
\nabla \mathrm{f}=\frac{\partial f}{\partial w_{i, j}}
$$

\section{Data preprocessing techniques}

The real-world data are mostly incompatible with most of the machine learning algorithms. The data are primarily incomplete and inconsistent. The data preprocessing techniques enable to machine learning algorithms process the data for the creation of a predictive model. Moreover, these techniques usually increase the accuracy of the model. Therefore, the data must be cleansed from errors and transformed the raw data into a predefined format.

The aim of the article is an evaluation of data preprocessing techniques which are usually used for continuous values preprocessing. We focused primarily on the issue of missing data inserting and data normalization. The missing values can be classified as a frequently occurring phenomenon in real-world applications. There are a considerable number of techniques which are trying to solve this issue. The most simplistic approach is to exclude all missing values from data. However, this approach could lead to an enormous loose amount of information. Moreover, the missing values could represent important information in case of anomaly detection field. A more common approach to solve this problem is to encode missing values into a numeric representation. There are several well-known inserting strategies for missing values. One of the basic inserting strategies replace the missing values with one static numeric values (zero is commonly used). The other strategy takes into consideration the distribution of values in every dataset attributes. For example, mean, a median of all values in the attribute.

The second chosen group of data preprocessing technique in the article is data normalization. These techniques are based on the assumption of the same scale for all attributes. All attributes in the dataset should have the same scale and hence the same weight. Moreover, the different scale could lead to misinterpretation of the attribute importance. The minmax scaling is a commonly used approach to solve this problem. However, there is a contradiction in what scale used for data normalization. The equation for the calculation of the new value of the attribute is shown in (5).

$$
x^{*}=\frac{x-\min }{\max -\min }\left(n e w \_m a x-n e w \_m i n\right)+n e w \_\min
$$

Where $\mathrm{x} *$ is a normalized value and $\mathrm{x}$ is an old value from a dataset. Variables min and max represent maximal or minimal values in the attribute. Variable new_max and new_min are new maximal and minimal values. The standardization is a second technique usually used for scaling the data. Standardization technique creates a dataset with zero mean and unit variance. The distribution of the dataset is centred near zero (6).

$$
x^{*}=\frac{x-\mu}{\delta}
$$

Where $\mathrm{x}^{*}$ is a standardized value and $\mathrm{x}$ is an old value from a dataset. The variable $\mu$ represents mean of attribute and $\delta$ represent the standard deviation of attribute.

\section{Methods}

We decide to implement a number of experiments in order to establish the best-suited approach for preprocessing continuous values in a dataset. Moreover, each experiment corresponds to a different setup for preprocessing techniques. Each setup is evaluated by datasets which include cyber attacks. The precisions of identification of anomalies (cyber attacks) will be determining factor. We create numerous autoencoders predictive model in order to evaluate the detection capability of each setup. Each model is evaluated according to several metrics: Accuracy, F1 Score, Specificity, Sensitivity, Precision, False positive rate and Time. In the case of the binary classification (normal behavior or anomal behavior), we often evaluate the accuracy of the model which represent a relationship between positive classification and all examples of classification. F1 score also known as F measure represents the ratio of precision and recall metrics which 
is called harmonic mean. Specificity metric measures the proportion of true identification of the negative class which is the normal behavior of the ICS network in this case. Sensitivity metric measures the proportion of true identification of the positive class which is represented as cyber attacks in this case. Precision metric measures the proportion between false and true positive class. The metric False positive rate (FPR) represents a rating of false to identify cyber attacks which can disrupt the availability of the ICS. The availability of services is the most important criteria for ICS systems. Therefore, FPR is one of the most crucial metrics for ICS. Each of these metrics is defined in rage from 0 to 1 where 0 is the worst situation and 1 is the best case of identification of anomalies. The last metric is Time which is needed to process dataset with a cyber attack.

The dataset presented in publication [10] is used to create predictive models and evaluate experiments. The authors provided pcap files which include cyber attacks and also records of regular network traffic of ICS. We extracted 19 numerical attributes in order to test and examine data preprocessing techniques. Each model is created based on a dataset which includes only records of the normal behavior of the ICS system. This dataset consists of 134690 records, where $70 \%$ of records are used to train the model, and the rest of $30 \%$ is used to validate the model. Moreover, three cyber attacks ("CnC uploading exe file", "6RTU with operate - exploit", "moving two files", sending fake command) were used to evaluate the detection capabilities of each model.

The six data preprocessing techniques (three techniques for handling missing values and three techniques for data normalization and standardization) are selected in order to change data representation. Moreover, we evaluate each possible setup in a total of nine options for each cyber attack. The selected method for replacement of missing values is based on attribute mean, median value or static replacement of number zero. We also establish standardization techniques and normalization technique (in the range 0,1 and -1,1) in order to transform data into a proper scale. Each of these techniques is fitted on normal dataset where the necessary representation is saved. Subsequently, each test dataset with a separated cyber attack is transformed according to saved representation. Therefore, the preprocessing processes of test datasets are not affected.

The presented approach based on autoencoder is based on regression. Therefore, the method to detect anomalies must be set. The presented anomaly detection system is based on the differentiation between a predicted data point and real values from the dataset. The first is the value predicted by the model $x_{i, j}^{\text {mod }}$, and the second is defined as real data from the dataset $x_{i, j}^{r e a l}$. Then the relationship can be defined as:

$$
x^{r}=\left|x_{i, j}^{\text {mod }}-x_{i, j}^{r e a l}\right|
$$

Where the value $\mathrm{x}$ belongs to the $\mathrm{i}$-th attribute at the $\mathrm{j}$-th data point. Moreover, the MSE formula is used to calculate the resulting deviation value for each data point, see (8). Furthermore, Precision/Recall curve was established in order to calculate the threshold between regular network traffic and malicious network traffic.

$$
\mathrm{MSE}=\frac{1}{2} \sum_{i=1}^{n}\left(x_{i}^{r}\right)^{2}
$$

\section{Results}

The article is focused on determining best-suited preprocessing techniques for network anomaly detection system in case of continuous values. Numerous experiments are designed in order to solve this problem. The general approach of machine learning depends on balanced training dataset. This approach is not possible in the case of anomaly detection. Moreover, the training dataset adopts only data from one class which represents regular behavior of the system. Therefore, there is a high probability of the occurrence of new values which are not presented in training dataset. This phenomenon also includes the presence of missing values. Moreover, each new and unique value can be characterized as an anomaly and therefore, can be a symptom of a cyber attack. 


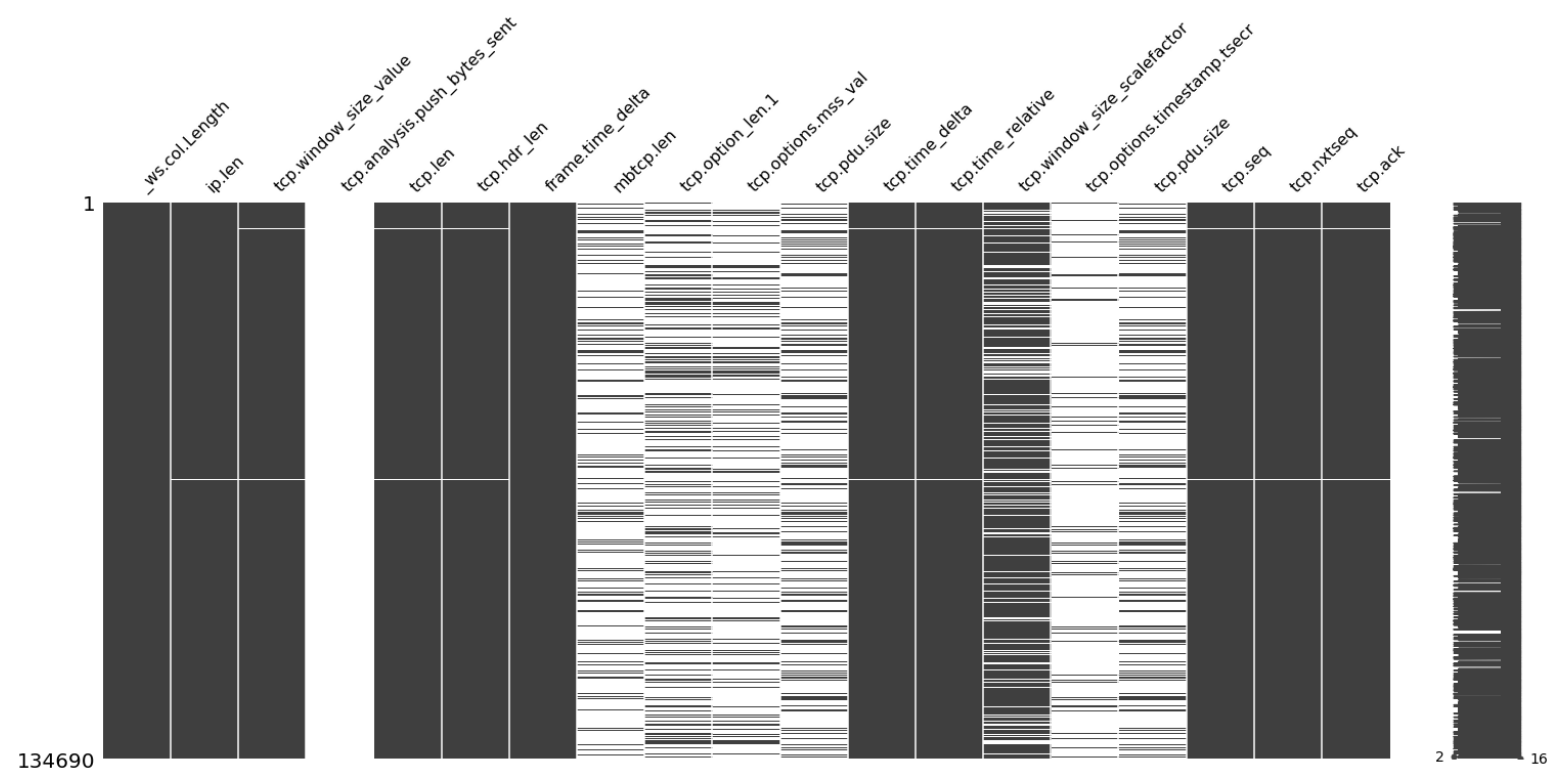

Fig. 1. Representation of missing values in the training dataset.

The representation of missing values in the training dataset is shown in Fig. 1, where is shown each attribute of the dataset. As can be seen in the figure, the attribute "tcp.analysis.push_bytes_sent" is only filled by missing values. However, the representation of the attribute is relatively different in comparison with the same attribute from test dataset (Fig. 2), where the cyber attack is present.

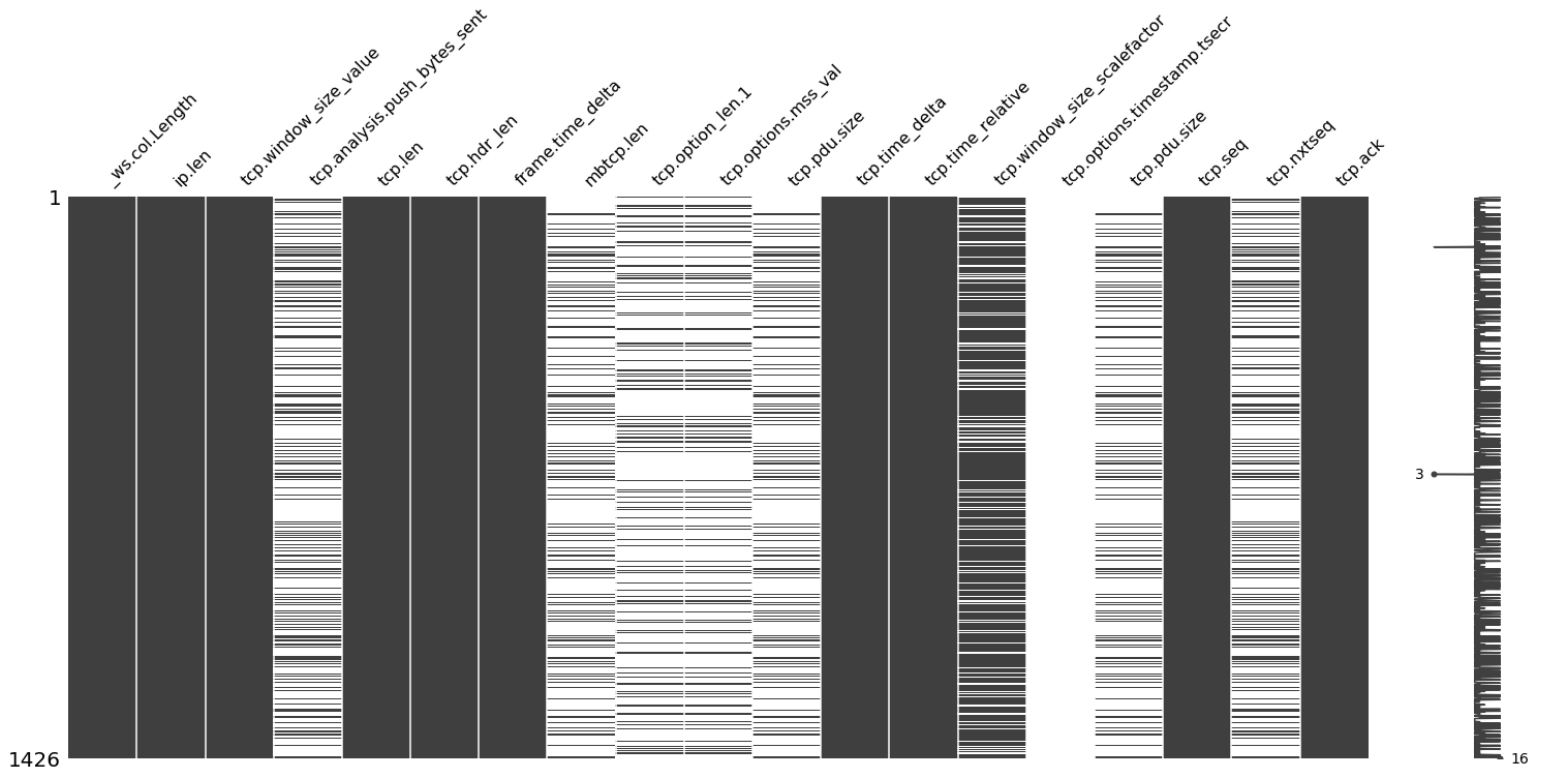

Fig. 2. Representation of missing values in the test dataset.

The difference between training and test dataset makes the attribute selection for anomaly detection little bit tricky. Thus, it is hard to tell which attributes are the most important and therefore will be included in the dataset and which attributes are least important for the anomaly detection. We decided to include presented nineteen attributees (Fig. 1, Fig. 2, Fig. 3) into datasets.

The analysis of the attributes in training dataset is made. Moreover, the heat map is utilized to define the relationship between individual attributes. The heat map represents the correlation between attributes. As can be seen in Fig. 3 there is a very weak linear relationship between attributes. 


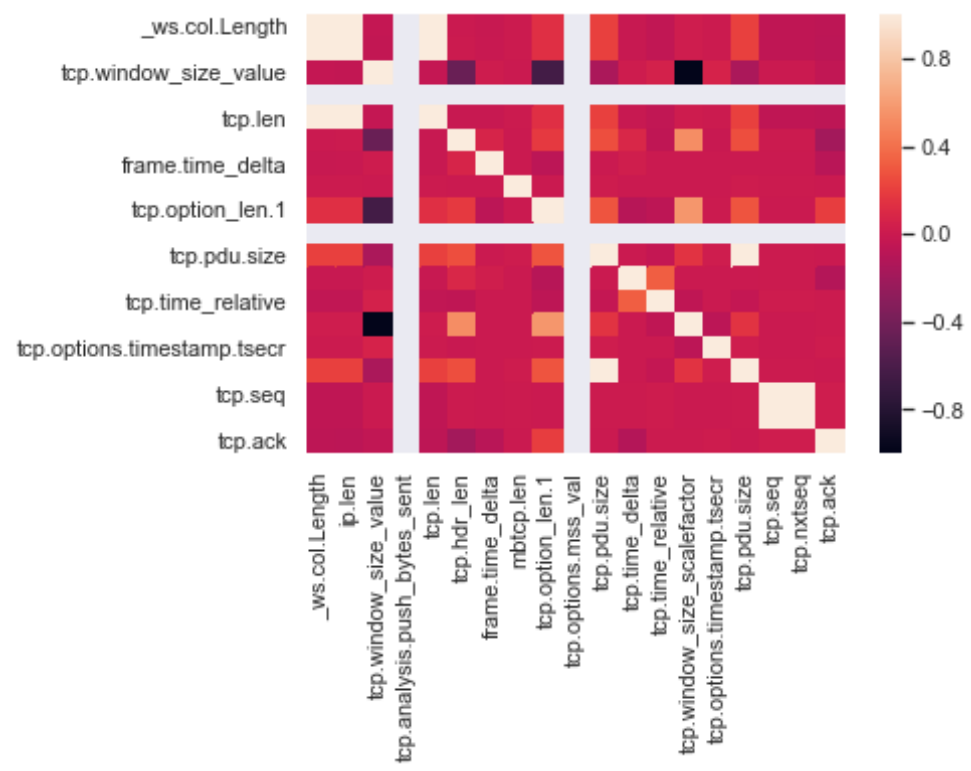

Fig. 3. Heat map of the training dataset.

The autoencoder setup was chosen as follow. Nesterov-accelerated Adaptive Moment Estimation was used as an optimization technique for loss function (Mean squared error) in the training procedure of the autoencoder. The autoencoder architecture is described in Fig. 4. Moreover, each model was trained for 200 epochs periods.

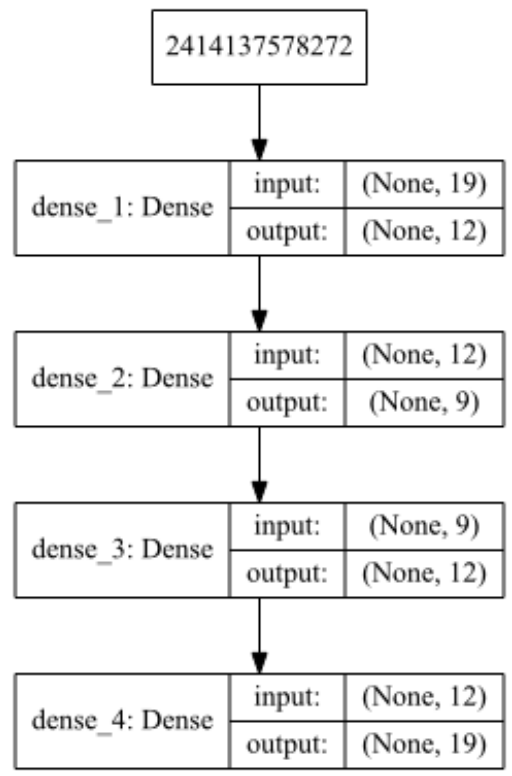

Fig. 4. Layers setup of the autoencoder.

The final result can be seen in table 1 and table 2. Each combination of preprocessing techniques was computed. Moreover, nine anomaly detection models were created. Each of the represent specific setup which is used to create models base on normal behavior of the ICS system. Each of the models was used to evaluate datasets with cyber attacks. We choose four cyber attacks with six metric and evaluation time in order to rate different preprocessing techniques.

\begin{tabular}{|c|c|c|c|c|c|c|c|c|c|c|c|c|c|c|}
\hline & \multicolumn{9}{|c|}{ CnC uploading exe file } & \multicolumn{4}{c|}{ 6RTU with operate - exploit } \\
\hline & Acc. & F1 score & Spec. & Sens. & Prec. & FPR & Time & Acc. & $\begin{array}{c}\text { F1 } \\
\text { score }\end{array}$ & Spec. & Sens. & Prec. & FPR & Time \\
\hline $\begin{array}{c}\text { mean; 0 to 1 } \\
\text { normalization }\end{array}$ & 0.9446 & 0.6722 & 0.9701 & 0.6694 & 0.675 & 0.0299 & 0.0105 & 0.4259 & 0.5557 & 0.189 & 0.5555 & 0.5559 & 0.811 & 0.0113 \\
\hline $\begin{array}{c}\text { mean; -1 to 1 } \\
\text { normalization }\end{array}$ & 0.9446 & 0.6722 & 0.9701 & 0.6694 & 0.675 & 0.0299 & 0.0103 & 0.6739 & 0.7476 & 0.5396 & 0.7473 & 0.7479 & 0.4604 & 0.0114 \\
\hline
\end{tabular}




\begin{tabular}{|c|c|c|c|c|c|c|c|c|c|c|c|c|c|c|}
\hline $\begin{array}{c}\text { mean; } \\
\text { standartization }\end{array}$ & 0.9446 & 0.6722 & 0.9701 & 0.6694 & 0.675 & 0.0299 & 0.0099 & 0.6189 & 0.705 & 0.4619 & 0.7048 & 0.7053 & 0.5381 & 0.0116 \\
\hline $\begin{array}{c}\text { median; 0 to 1 } \\
\text { normalization }\end{array}$ & 0.9446 & 0.6722 & 0.9701 & 0.6694 & 0.6750 & 0.0299 & 0.0100 & 0.6491 & 0.7284 & 0.5046 & 0.7281 & 0.7287 & 0.4954 & 0.0140 \\
\hline $\begin{array}{c}\text { median; -1 to 1 } \\
\text { normalization }\end{array}$ & 0.9446 & 0.6722 & 0.9701 & 0.6694 & 0.6750 & 0.0299 & 0.0111 & 0.6135 & 0.7009 & 0.4543 & 0.7006 & 0.7012 & 0.5457 & 0.0144 \\
\hline $\begin{array}{c}\text { median; } \\
\text { standartization }\end{array}$ & 0.9446 & 0.6722 & 0.9701 & 0.6694 & 0.6750 & 0.0299 & 0.0101 & 0.5747 & 0.6708 & 0.3994 & 0.6706 & 0.6711 & 0.6006 & 0.0112 \\
\hline $\begin{array}{c}\text { constant; 0 to 1 } \\
\text { normalization }\end{array}$ & 0.9446 & 0.6722 & 0.9701 & 0.6694 & 0.6750 & 0.0299 & 0.0106 & 0.5736 & 0.6700 & 0.3979 & 0.6697 & 0.6703 & 0.6021 & 0.0120 \\
\hline $\begin{array}{c}\text { constant; -1 to 1 } \\
\text { normalization }\end{array}$ & 0.9446 & 0.6722 & 0.9701 & 0.6694 & 0.6750 & 0.0299 & 0.0101 & 0.6609 & 0.7376 & 0.5213 & 0.7373 & 0.7379 & 0.4787 & 0.0115 \\
\hline $\begin{array}{c}\text { constant; } \\
\text { standartization }\end{array}$ & 0.9446 & 0.6722 & 0.9701 & 0.6694 & 0.6750 & 0.0299 & 0.0085 & 0.6555 & 0.7334 & 0.5137 & 0.7331 & 0.7337 & 0.4863 & 0.0122 \\
\hline
\end{tabular}

Table 1. The results of preprocessing techniques for first two cyber attacks.

\begin{tabular}{|c|c|c|c|c|c|c|c|c|c|c|c|c|c|c|}
\hline & \multicolumn{9}{|c}{ Moving two files } & \multicolumn{1}{c|}{ Sending fake command } \\
\hline & Acc. & F1 score & Spec. & Sens. & Prec. & FPR & Time & Acc. & $\begin{array}{c}\text { F1 } \\
\text { score }\end{array}$ & Spec. & Sens. & Prec. & FPR & Time \\
\hline $\begin{array}{c}\text { mean; 0 to 1 } \\
\text { normalization }\end{array}$ & 0.9605 & 0.1208 & 0.98 & 0.12 & 0.1216 & 0.02 & 0.0202 & 0.8766 & 0 & 0.8774 & 0 & 0 & 0.1226 & 0.0592 \\
\hline $\begin{array}{c}\text { mean; -1 to 1 } \\
\text { normalization }\end{array}$ & 0.9792 & 0.5369 & 0.9895 & 0.5333 & 0.5405 & 0.0105 & 0.0406 & 0.9723 & 0 & 0.9732 & 0 & 0 & 0.0268 & 0.0568 \\
\hline $\begin{array}{c}\text { mean; } \\
\text { standartization }\end{array}$ & 0.9557 & 0.0134 & 0.9775 & 0.0133 & 0.0135 & 0.0225 & 0.0409 & 0.8822 & 0 & 0.883 & 0 & 0 & 0.1167 & 0.0681 \\
\hline $\begin{array}{c}\text { median; 0 to 1 } \\
\text { normalization }\end{array}$ & 0.9587 & 0.0805 & 0.9790 & 0.0800 & 0.0811 & 0.0210 & 0.0448 & 0.8773 & 0 & 0.8781 & 0 & 0 & 0.1219 & 0.0597 \\
\hline $\begin{array}{c}\text { median; -1 to 1 } \\
\text { normalization }\end{array}$ & 0.9569 & 0.0403 & 0.9781 & 0.0400 & 0.0405 & 0.0219 & 0.0466 & 0.9664 & 0 & 0.9673 & 0 & 0 & 0.0327 & 0.0595 \\
\hline $\begin{array}{c}\text { median; } \\
\text { standartization }\end{array}$ & 0.9563 & 0.0268 & 0.9778 & 0.0267 & 0.0270 & 0.0222 & 0.0407 & 0.8732 & 0 & 0.8740 & 0 & 0 & 0.1260 & 0.0567 \\
\hline $\begin{array}{c}\text { constant; 0 to 1 } \\
\text { normalization }\end{array}$ & 0.9599 & 0.1074 & 0.9796 & 0.1067 & 0.1081 & 0.0204 & 0.0440 & 0.9721 & 0 & 0.9729 & 0 & 0 & 0.0271 & 0.0726 \\
\hline $\begin{array}{c}\text { constant; -1 to 1 } \\
\text { normalization }\end{array}$ & 0.9726 & 0.3893 & 0.9861 & 0.3867 & 0.3919 & 0.0139 & 0.0414 & 0.9506 & 0 & 0.9515 & 0 & 0 & 0.0485 & 0.0572 \\
\hline $\begin{array}{c}\text { constant; } \\
\text { standartization }\end{array}$ & 0.9599 & 0.1074 & 0.9796 & 0.1067 & 0.1081 & 0.0204 & 0.0458 & 0.9452 & 0 & 0.9460 & 0 & 0 & 0.0540 & 0.0673 \\
\hline
\end{tabular}

Table 2. The results of preprocessing techniques for last two cyber attacks.

Each row represents a combination of preprocessing techniques. The first technique for preprocessing of missing values and the second for the scale change of the data representation. The columns represent evaluation metrics (Accuracy, F1 Score, Specificity, Sensitivity, Precision, False positive rate (FPR) and Time) where Accuracy, F1 Score, Specificity, Sensitivity, Precision should be maximized and FPR and Time should be minimized. The cyber attack "CnC uploading exe file" seems to be unaffected by preprocessing techniques. Except for prediction time which is lowest for the combination of constant (replacement of missing values with 0) and standardization technique. The detection capabilities of the other model are changing based on preprocessing techniques. Except for cyber attack "Sending fake command" where the metric F1 score, Sensitivity and Precision acquired zero values.

\section{Conclusion}

The aim of the article is focused on the cyber security of the ICS systems. The network attributes were considered as the primal information to detect cyber attacks. Moreover, the anomaly detection algorithm was adopted to detect anomalies as the symptom of cyber attacks. The main question which should be answered: which preprocessing techniques are optimal for anomaly detection task in the ICS network environment? Therefore, two main groups of the preprocessing techniques were considered which are responsible for handling missing values and scale of the data. There were established nine combinations of selected techniques. The created models were evaluated by four datasets which include cyber attacks.

The experiment includes only continuous numerical values. Thus, only a limited part of the data was considered. The categorical data represent the bigger part of the network communication. Therefore, the results were relatively poor. This fact supported the heat map in Fig. 3 where low linear relationships between attributes were shown. However, we decide to isolate continuous data from categorical in order to evaluate preprocessing techniques which would be difficult with the whole dataset. Fig. 1 and Fig. 2 shows how difficult attribute(feature) selection could be in the case of anomaly detection. There should be considered all possible attributes in dataset in order to cover all possibilities and all aspects of anomalies. 
The results include four cyber attacks. However, one of them ( $\mathrm{CnC}$ uploading exe file) did not exhibit any change of metrics values based on preprocessing techniques combinations. Therefore, a cyber attack could be excluded. The overall best results were achieved by a combination of mean value replacement and normalization from -1 to 1 . This combination of preprocessing techniques achieve the best results in all evaluation metrics except prediction time. However, the difference between the individual combination of preprocessing techniques is relatively insignificant; therefore, the prediction time is unimportant metric in this case. Furthermore, the second and third-best score belongs to the following combinations(median; -1 to 1 normalization and constant; - 1 to 1 normalization). The conclusion of the results is quite surprising. The preprocessing techniques for replacement of missing values are insignificant. Moreover, the value scaling of the dataset can have a considerable impact on the detection capabilities of the anomaly detection system. In our case, the most suitable choice is considered rescaling the dataset in the range from -1 to 1 .

We investigated the capabilities of preprocessing techniques in the anomaly detection environment. The future research should include the preprocessing of categorical data which can be quite considerable difficult. Especially in case of categorical data of high cardinality.

\section{Acknowledgments}

This work was funded by the Internal Grant Agency (IGA/FAI/2019/002) and supported by the research project VI20172019054 "An analytical software module for the real-time resilience evaluation from point of the converged security ", supported by the Ministry of the Interior of the Czech Republic in the years 2017-2019. Finally, we thank Lemay and Fernandez [10] who provides ICS datasets.

\section{References}

[1] Kučak, D., Juričić, V., \& Đambić, G. (2018). Machine Learning in Education-A Survey of Current Research Trends. Annals of DAAAM \& Proceedings, 29.

[2] Aleksandr, F., \& Vyacheslav, S. (2018). Recommendation System Based on Neural Network for Prediction of Emergency Situations. Annals of DAAAM \& Proceedings, 29.

[3] Stouffer, K., Lightman, S., Pillitteri, V., Abrams, M., \& Hahn, A. (2015). Guide to industrial control systems (ICS) security. NIST special publication, 800(82) R2, 16-16.

[4] Rajendran, S., Lenders, V., Meert, W., \& Pollin, S. (2019). Crowdsourced wireless spectrum anomaly detection. arXiv preprint arXiv:1903.05408.

[5] Ruff, L., Vandermeulen, R. A., Görnitz, N., Binder, A., Müller, E., Müller, K. R., \& Kloft, M. (2019). Deep SemiSupervised Anomaly Detection. arXiv preprint arXiv:1906.02694.

[6] Camacho, J., Maciá-Fernández, G., Fuentes-García, N. M., \& Saccenti, E. (2019). Semi-supervised multivariate statistical network monitoring for learning security threats. IEEE Transactions on Information Forensics and Security, 14(8), 2179-2189.

[7] García, S., Luengo, J., \& Herrera, F. (2015). Data preprocessing in data mining (pp. 59-139). New York: Springer.

[8] García, S., Ramírez-Gallego, S., Luengo, J., Benítez, J. M., \& Herrera, F. (2016). Big data preprocessing: methods and prospects. Big Data Analytics, 1(1), 9.

[9] Davis, J. J., \& Clark, A. J. (2011). Data preprocessing for anomaly based network intrusion detection: A review. computers \& security, 30(6-7), 353-375.

[10] Lemay, A., \& Fernandez, J. M. (2016) Providing \{SCADA\} Network Data Sets for Intrusion Detection Research. In: 9th Workshop on Cyber Security Experimentation and Test (\{CSET $\} 16)$. 